\title{
Efficacy of adalimumab for the treatment of extraintestinal manifestations of Crohn's disease
}

\author{
Manuel Barreiro-de-Acosta ${ }^{1,2}$, Aurelio Lorenzo ${ }^{1}$ and J. Enrique Domínguez-Muñoz ${ }^{1,2}$ \\ ${ }^{1}$ Department of Gastroenterology. ${ }^{2}$ Foundation for Research in Digestive Diseases (FIENAD). \\ Hospital Universitario de Santiago de Compostela. A Coruña, Spain
}

\begin{abstract}
Background and aims: Crohn's disease (CD) is commonly associated with inflammatory processes located in organs and systems others than the gut, which are known as extraintestinal manifestations (EIM) of the disease. The aim of this study was to assess the effectiveness of adalimumab (ADA) for the treatment of EIM in patients with CD.

Methods: forty two consecutive $\mathrm{CD}$ patients with at least one EIM were prospectively included in a open-label study. Patients received ADA (160 mg at week zero, $80 \mathrm{mg}$ at week two and 40 mg every other week) over six months and the effectiveness and safety of ADA for EIMs were assessed. The influence of gender, age, smoking habits, family history of inflammatory bowel disease, phenotype and previous anti-TNF treatment on EIM resolution was also investigated.

Results: at month six, $76.2 \%$ of the patients showed remission or response in $\mathrm{CD}$ (33.3\% remission and $42.9 \%$ any response). EIM showed a parallel course with CD in most cases, and showed remission or response in $66.7 \%$ of patients (38.1\% remission and $28.5 \%$ any response). Patients with any response of their EIM condition were younger than those with no response $(p=0.04)$. No relationship was found between sex, tobacco, family history of IBD, phenotype and previous treatment with anti-TNF, and EIM resolution.

Conclusions: adalimumab is effective in reducing EIM of CD. Age but not tobacco, CD phenotype and anti-TNF-naïve status appears to influence the response.
\end{abstract}

Key words: Adalimumab. Extraintestinal manifestations. AntiTNF.

Barreiro-de-Acosta M, Lorenzo A, Domínguez-Muñoz JE. Efficacy of adalimumab for the treatment of extraintestinal manifestations of Crohn's disease. Rev Esp Enferm Dig 2012;104:468-472.

Received: $13-04-2012$

Accepted: $10-09-2012$

Correspondence: Manuel Barreiro-de Acosta. Department of Gastroenterology. Hospital Universitario de Santiago de Compostela. C/ Choupana, s/n. 15706 Santiago de Compostela. A Coruña, Spain

e-mail: manubarreiro@hotmail.com

\section{INTRODUCTION}

Crohn s disease (CD) is a chronic inflammatory disorder of the digestive tract that mainly involves ileum and colon. Between $20-40 \%$ of the patients with CD develop chronic inflammatory conditions located in other organs and systems, which are known as extraintestinal manifestations (EIM) of the disease (1-9). EIM are associated with an additional and significant morbidity and impaired quality of life (10). The most common EIM affect the joints, skin, eyes and biliary tract (11). Peripheral arthropathy (pauciarticular, asymmetrical and migratory) occurs in 10-20\% of inflammatory bowel disease (IBD) patients and is characterized by a recurrent nature and a parallel course with CD (1113). In contrast, axial arthropathy shows an independent course of $\mathrm{CD}$ and includes sacroiliitis, with a prevalence of $12-25 \%$, and ankylosing spondylitis, with a prevalence of $2-16 \%$ in Crohn's patients (11-15).

Skin manifestations, such as erythema nodosum (EN) and pyoderma gangrenosum (PG), appear respectively in $3-20 \%$ and $0.5-20 \%$ of Crohn's patients. PG is more severe than $\mathrm{EN}$, and is associated with pustules, nodules and ulcers (11). EN onset is related with the onset of CD and its remission is parallel to $\mathrm{CD}$ improvement. However, $\mathrm{PG}$ is a more aggressive form and does not always improve in parallel with $\mathrm{CD}(13,16)$.

Ophthalmologic disorders mainly episcleritis and uveitis are less frequent and range from 2 to $6 \%$ of IBD patients. While episcleritis is a painless condition, uveitis is related with pain, blurry vision and photophobia, leading to blindness in chronic and untreated cases. Similarly to axial arthropathy and PG, uveitis is not always associated to active $\operatorname{CD}(8,11,13)$.

Crohn's disease treatment is targeted to reduce inflammation with corticosteroids, immunosupressants and tumor necrosis factor (TNF) antagonists (17). Adalimumab (ADA) is a human monoclonal anti-TNF that has demonstrated efficacy in inducing and maintaining remission in patients 
with moderate to severe CD. ADA is effective not only in naïve patients but also in patients with loss of response or intolerance to infliximab (IFX) (chimeric monoclonal antiTNF) (18-22). Although, EIM evolution is not parallel to $\mathrm{CD}$, most EIM respond to anti-TNF treatment. After 20 weeks of treatment in CARE study, $79 \%$ of patients had resolution of at least one EIM and 51\% were free of any sign and symptom (23).

Efficacy of ADA for the treatment of EIM in CD has not been investigated in depth. The aim of this study was to assess the effectiveness of ADA for the treatment of EIM in patients with $\mathrm{CD}$. Additionally, the relationship between the effectiveness of ADA and well known potential risk factors of the disease as smoking and family history of IBD was assessed. Gender, age, CD phenotype and previous treatment with anti-TNF were also evaluated as independent variables potentially influencing the effectiveness of ADA.

\section{MATERIALS AND METHODS}

The study was designed as an open-label, prospective and consecutive, single centre study. Patients older than 18 years with moderate to severe CD and any active EIM attending to the Inflammatory Bowel Disease Unit of the University Hospital of Santiago de Compostela from 2007 to 2010 were invited to the study. All patients were Caucasian and from Galicia (North-West of Spain). CD was diagnosed based on the clinical picture, physical examination, ileocolonoscopy with biopsies for histological confirmation and barium radiology, magnetic resonance and enteroclysis for extension study. Patients were included if they presented a Crohn's Disease Activity Index (CDAI) over 200 points.

Clinical history was reviewed and patients were interviewed for sex, age, smoking habits, family history of inflammatory bowel disease and previous anti-TNF therapy. A positive family history of inflammatory bowel disease was defined as the presence of at least one first-degree relative suffering from the disease.

Participants were examined for the main reactive and non-reactive EIM of CD. Reactive conditions were defined as those related to acute gut inflammation, among them dermatologic lesions (erythema nodosum and pyoderma gangrenosum), major eye complications (episcleritis and uveitis) and peripheral osteoarthritis.

Arthritis was defined as the presence of joint swelling or effusion. Patients without an evidence of swelling were classified as arthralgia, which was not included as EIM of CD. Sacroiliitis was confirmed by radiology in patients who presented characteristic symptoms as inflammatory low back pain or buttock pain. Dermatologic, rheumatologic and ophthalmologic manifestations were confirmed by the corresponding specialist.

Non-reactive conditions of EIM associated with $\mathrm{CD}$ were defined as those unrelated to gut inflammation, such as ankylosing spondylitis, sacroiliitis and sclerosing cholan- gitis. Extraintestinal manifestations related with a longstanding inflammatory bowel disease, such as anaemia, as well as drug-related manifestations, as pancreatitis and neutropenia, were excluded.

Patients included in the study were treated with subcutaneous ADA. Dosage regiment consisted in an initial dose of $160 \mathrm{mg}$, a dose of $80 \mathrm{mg}$ at week 2 and maintenance dose of $40 \mathrm{mg}$ every other week during 6 months of treatment. In case of lost of response, a dose escalation, defined as $40 \mathrm{mg}$ every week, was proposed.

$\mathrm{CD}$ and EIM were clinically evaluated by two independent gastroenterologists at month 0 and 6 . Patients were classified as presenting remission, partial response and non-response. Remission of CD symptoms were considered when CDAI $<150$ points and response when a decrease over 75 points was achieved in CDAI measured in month 0 .

For EIM outcome classification, remission was defined as the total absence of EIM symptoms. Partial response was defined as improvement of the EIM symptoms in comparison with week 0. Major eye complications diagnosis was confirmed with an independent ophthalmologist. There was consensus in the classification of CD and EIM evolution between every of the specialist involved in all cases.

\section{Statistical analysis}

Categorical variables collected in the study are shown as percentages, and were analyzed by the chi-square test or the Fisher exact test as appropriate. The potential influence of gender, tobacco, anti-TNF-naïve status and CD phenotype according to the Montreal Classification on the efficacy of ADA therapy for EIM was analyzed by unconditional stepwise logistic regression analysis. Response of EIM to ADA therapy was considered as a binary outcome for logistic analysis: any response (remission or partial response) or no response.

\section{RESULTS}

Forty-two patients with $\mathrm{CD}$ and active extraintestinal manifestations were included in the study; 22 males (52\%) and 20 females (48\%). Mean age was 38 years, ranged from 20 to 56 years. According to the Montreal classification, $21(50 \%)$ patients presented the B1 phenotype (non-stricturing non-penetrating) and the other 21 patients (50\%) B3 phenotype (penetrating) (24).

Two patients $(5 \%)$ declared a family history of IBD. Exposure to tobacco, a known risk factor for IBD, was referred in $14(33 \%)$ of patients, while $16(38.1 \%)$ were non smokers and $12(28.6 \%)$ ex-smokers. Half of the patients were previously treated with IFX and $26(62 \%)$ were concomitantly treated with azathioprine.

Peripheral arthritis appeared in 31 (73.8\%) of participants. Seven cases $(16.7 \%)$ presented ankylosing spondylitis and two $(4.8 \%)$ pyoderma grangrenosum. Cases of 
Table I. Response of different EIM to ADA therapy

\begin{tabular}{lll}
\hline EIM & $\begin{array}{l}\text { Partial response or } \\
\text { remission }\end{array}$ & No response \\
\hline Peripheral arthritis & 19 & 12 \\
Sacroiliitis & 1 & 0 \\
Ankylosing spondylitis & 5 & 2 \\
Uveitis & 1 & 0 \\
Pyoderma grangrenosum & 2 & 0 \\
\hline
\end{tabular}

sacroiliitis and uveitis were infrequent and only were detected in one patient respectively.

At month 6, CD showed remission in $33.3 \%$ of patients, response in $42.8 \%$ and no response in $23.9 \%$. Tobacco, previous treatment with IFX or CD phenotype did not influence the therapeutic response to ADA. Six patients (14.2\%) needed dose escalation and 4 patients $(9.4 \%)$ did not complete ADA therapy: $2(4.7 \%)$ because of adverse events related to ADA (dermatologic reactions) and $2(4.7 \%)$ due to the absence of $\mathrm{CD}$ response after 10 weeks.

At month six, $38.1 \%$ of the patients showed a complete remission of the EIM and $28.5 \%$ experienced partial response (global response to ADA of $66.6 \%$ of EIM). The efficacy of ADA for individual EIM of CD is shown in table I. The influence of the different variables evaluated in the effectiveness of ADA for EIM is shown in table II. Patients with EIM responding to ADA were younger than those with no response $(\mathrm{p}=0.04)$. Gender, smoking habits, phenotype and previous anti-TNF therapy had no influence on the efficacy of ADA for EIM of CD. Response of EIM to ADA correlated with response of $\mathrm{CD}(\mathrm{rho}=0.703, \mathrm{p}=0.001)$ and $85.7 \%$ of patients were classified in the same group (response or no response) in both outcomes.

\section{DISCUSSION}

The present study shows that ADA is effective for the therapy of EIM in CD patients. In fact, two out of each three patients with EIM of CD benefit from ADA therapy. Previous reports of the effectiveness of ADA in the remission of EIM of CD are scarce. ADA has demonstrated to be effective to induce and maintain remission in patients with active $C D$ either naïve to biologic agents or with prior loss of response or intolerance to IFX. However, additional benefits of the treatment and the influence of different fac-

Table II. Influence of clinical characteristics in the global response of EIM to ADA

\begin{tabular}{|c|c|c|c|c|}
\hline & \multicolumn{4}{|c|}{ Global response of EIM } \\
\hline & & Partial response or remmision & No response & \\
\hline \multicolumn{2}{|l|}{ Categorical variables } & $n(\%)$ & $n(\%)$ & $p\left(\operatorname{Pr}>c h i^{2}\right)$ \\
\hline \multicolumn{5}{|l|}{ Sex } \\
\hline \multicolumn{5}{|l|}{ Naïve } \\
\hline & Yes & $16(76,2 \%)$ & $5(23,8 \%)$ & 0.190 \\
\hline & No & $12(57,1 \%)$ & $9(42,8 \%)$ & \\
\hline \multicolumn{5}{|c|}{ Crohn's response to ADA } \\
\hline & Remission & $13(92,8 \%)$ & $1(7,1 \%)$ & 0.001 \\
\hline & Response & $14(77,7)$ & $4(22,2 \%)$ & \\
\hline & No & $25(69.4 \%)$ & $11(30.5 \%)$ & \\
\hline \multicolumn{5}{|l|}{ Crohn's behaviour (B) } \\
\hline & Inflammatory (B1) & $15(71.4 \%)$ & $6(28.5 \%)$ & 0.512 \\
\hline & Penetrating (B3) & $13(61.9 \%)$ & $8(38.1 \%)$ & \\
\hline \multicolumn{5}{|l|}{ Tobacco } \\
\hline & Smoker & $8(57.1 \%)$ & $6(42.8 \%)$ & 0.334 \\
\hline & Non-smoker & $11(68.7 \%)$ & $5(31.2 \%)$ & \\
\hline & Ex-smoker & $9(75 \%)$ & $3(25 \%)$ & \\
\hline \multicolumn{2}{|l|}{ Continous variables } & Mean $\pm S D$ & Mean (SD) & $p(T<t)$ \\
\hline \multicolumn{2}{|l|}{ Age } & $36.57 \pm 1.88$ & $42 \pm 7.54$ & 0.040 \\
\hline
\end{tabular}


tors in the improvement of EIM remain not fully understood $(18-20,25)$.

The present study showed a high rate of CD response (remission or partial response) to ADA compared with previous studies with different dosages or follow-up time (1820,25). Three out of each four CD patients responded either completely or partially to ADA therapy. Six month treatment period with $40 \mathrm{mg}$ ADA every other week after induction doses of 160 and $80 \mathrm{mg}$ in the first two weeks seems to be adequate to achieve high response rates with a low number of withdrawals associated with adverse outcomes. Although some studies pointed out an inverse relationship between response rate and number of cigarettes (25) or prior treatment with IFX (21), our study and others showed no differences in Crohn's response to ADA in smokers $(9,18,20)$ and previous IFX treatment $(20,25)$.

Limited data about the efficacy of ADA on EIM resolution have been previously reported $(21,26,27)$. CARE study was the largest published study assessing the efficacy of ADA on EIM resolution with near one thousand patients included (21). Results from that study showed 51\% of patients free of any sign or symptom of EIM at week 20 with similar dosages than used in our study (21). Peripheral arthritis appears to be the most frequent EIM of CD. Response or remission was achieved in $61.3 \%$ patients with peripheral arthritis in present study, similar to the efficacy reported with IFX (28), and inferior to the $75 \%$ efficacy obtained with ADA in the CARE study. Although the design of these studies does not allow a face to face comparison of the results, peripheral arthritis appears to have an adequate response to ADA.

Regarding ankylosing spondylitis, response was achieved in $71.4 \%$ patients. A clinical trial assessing the efficacy and safety of ADA in patients with ankylosing spondylitis concluded that $58 \%$ of patients who received $40 \mathrm{mg}$ of antibody every other week achieved improvement, based in the Ankylosing Spondylitis International Working Group criteria (ASAS20) (29). CARE study (21), however, found similar number of patients ankylosing spondylitis at week zero compared with week 20 (16 vs. 15) with the same doses than in our study. Other EIM were present in isolated cases in the present study and conclusions about the efficacy of ADA cannot be drawn.

We found that patients with prior loss of response or intolerance to IFX presented similar response than naïve patients to ADA in CD and EIM symptoms, as it has been described in previous studies $(20,21,25)$. CHARM clinical trial described higher rates of remission of CD symptoms in patients treated with ADA and naïve to previous antiTNF drugs compared with those previously exposed to antiTNF therapies, but differences were not statistically significant (20). CARE study also found similar resolution rates regardless of prior IFX use, and were similar in patients with previous non-response to IFX and those who discontinued the therapy due to other reasons (21).

The present study shows that gender, smoking habits or phenotype of CD have not influence in the response of EIM to ADA, which supports previously reported data $(9,18,20,21)$. However, efficacy of ADA for the therapy of EIM of CD is influenced by age, and young patients showed a better response than older ones. Some authors pointed as a possible explanation of EIM the alterations of the tissue integrity by intestinal inflammatory conditions and the loss of barrier function that could allow the flow of substances (bacterial and food components) out into the systemic circulation and the activation of the immune system (30). This loss of integrity may impair with age while the immune system grow more reactive and cumulated damage in tissues may decrease the response of EIM to ADA.

In spite of the limitations of this study, including the open-label and uncontrolled design, and the relative low number of patients included with an over-representation of patients with peripheral arthritis, our results add relevant evidence in a topic in which the reported data are scarce. Our data support previous studies, which required confirmation in different settings.

In conclusion, ADA shows a high effectiveness in reducing extraintestinal manifestations of CD. Age but not tobacco, CD phenotype and anti-TNF-naïve status appears to influence the response.

\section{REFERENCES}

1. Bernstein CN, Blanchard JF, Rawsthorne P, Yu N, The prevalence of extraintestinal diseases in inflammatory bowel disease: a populationbased study. Am J Gastroenterol 2001;96:1116-22.

2. Greenstein AJ, Janowitz HD, Sachar DB. The extra-intestinal complications of Crohn's disease and ulcerative colitis: a study of 700 patients. Medicine (Baltimore) 1976;55:401-12.

3. Lakatos L, Pandur T, David G, Balogh Z, Kuronya P, Tollas A, et al. Association of extraintestinal manifestations of inflammatory bowel disease in a province of western Hungary with disease phenotype: results of a 25-year follow-up study. World J Gastroenterol 2003;9:2300-7.

4. Larsen S, Bendtzen K, Nielsen $\mathrm{OH}$. Extraintestinal manifestations of inflammatory bowel disease: epidemiology, diagnosis, and management. Ann Med 2010;42:97-114.

5. Rankin GB, Watts HD, Melnyk CS, Kelley ML, Jr. National Cooperative Crohn's Disease Study: extraintestinal manifestations and perianal complications. Gastroenterology 1979;77:914-20.

6. Ricart E, Panaccione R, Loftus EV, Tremaine WJ, Harmsen WS, Zinsmeister AR, et al. Autoimmune disorders and extraintestinal manifestations in first-degree familial and sporadic inflammatory bowel disease: a case-control study. Inflamm Bowel Dis 2004;10:207-14.

7. Salvarani C, Vlachonikolis IG, van der Heijde DM, Fornaciari G, Macchioni P, Beltrami M, et al. Musculoskeletal manifestations in a population-based cohort of inflammatory bowel disease patients. Scand J Gastroenterol 2001;36:1307-13.

8. Veloso FT, Carvalho J, Magro F. Immune-related systemic manifestations of inflammatory bowel disease. A prospective study of 792 patients. J Clin Gastroenterol 1996;23:29-34.

9. Barreiro-de Acosta M, Dominguez-Munoz JE, Nunez-Pardo de Vera MC, Lozano-Leon A, Lorenzo A, Pena S. Relationship between clinical features of Crohn's disease and the risk of developing extraintestinal manifestations. Eur J Gastroenterol Hepatol 2007;19:73-8.

10. Van Assche G, Dignass A, Reinisch W, van der Woude CJ, Sturm A, De Vos M, et al. The second European evidence-based Consensus on the diagnosis and management of Crohn's disease: Special situations. J Crohns Colitis 2010;4:63-101.

11. Barrie A, Regueiro M. Biologic therapy in the management of extraintestinal manifestations of inflammatory bowel disease. Inflamm Bowel Dis 2007; 13:1424-9. 
12. Repiso A, Alcántara M, Muñoz-Rosas C, Rodríguez-Merlo R, PérezGrueso MJ, Carrobles JM, et al. Extraintestinal manifestations of Crohn's disease: prevalence and related factors. Rev Esp Enferm Dig 2006;98:510-7.

13. Su CG, Judge TA, Lichtenstein GR. Extraintestinal manifestations of inflammatory bowel disease. Gastroenterol Clin North Am 2002; 31:307-27.

14. De Vos M. Joint involvement associated with inflammatory bowel disease. Dig Dis 2009;27:511-5.

15. Salvarani C, Fries W. Clinical features and epidemiology of spondyloarthritides associated with inflammatory bowel disease. World J Gastroenterol 2009;15:2449-55.

16. Engel MA, Neurath MF. New pathophysiological insights and modern treatment of IBD. J Gastroenterol 2010;45:571-83.

17. Podolsky DK. Inflammatory bowel disease. N Engl J Med 2002; 347:417-29.

18. Hanauer SB, Sandborn WJ, Rutgeerts P, Fedorak RN, Lukas M, MacIntosh $\mathrm{D}$, et al. Human anti-tumor necrosis factor monoclonal antibody (adalimumab) in Crohn's disease: the CLASSIC-I trial. Gastroenterology 2006;130:323-33.

19. Sandborn WJ, Rutgeerts P, Enns R, Hanauer SB, Colombel JF, Panaccione R, et al. Adalimumab induction therapy for Crohn disease previously treated with infliximab: a randomized trial. Ann Intern Med 2007:146:829-38

20. Colombel JF, Sandborn WJ, Rutgeerts P, Enns R, Hanauer SB, Panaccione $\mathrm{R}$, et al. Adalimumab for maintenance of clinical response and remission in patients with Crohn's disease: the CHARM trial. Gastroenterology 2007;132:52-65.

21. Lofberg R, Louis EV, Reinisch W, Robinson AM, Kron M, Camez A, et al. Adalimumab produces clinical remission and reduces extraintestinal manifestations in Crohn's disease: Results from CARE. Inflamm Bowel Dis 2012;18:1-9.

22. López Palacios N, Mendoza JL, Taxonera C, Lana R, Fuentes Ferrer M, Díaz-Rubio M. Adalimumab induction and maintenance therapy for Crohn's disease. An open-label study. Rev Esp Enferm Dig 2008:100:676-81.

23. Cordero Ruiz P, Castro Márquez C, Méndez Rufián V, Castro Laria L, Caunedo Álvarez A, Romero Vázquez J, et al. Efficacy of adalimumab in patients with Crohn's disease and failure to infliximab therapy: a clinical series. Rev Esp Enferm Dig 2011;103:294-8.

24. Satsangi J, Silverberg MS, Vermeire S, Colombel JF. The Montreal classification of inflammatory bowel disease: controversies, consensus, and implications. Gut 2006;55:749-53.

25. Triantafillidis JK, Mantzaris G, Karagiannis J, Papavasilliou E, Papatheodoridis G, Fouskas J, et al. Similar response to adalimumab in patients with active Crohn's disease either naive to biologic agents or with prior loss of response or intolerance to infliximab. Rev Med Chir Soc Med Nat Iasi 2010;114:85-90.

26. Karmiris K, Paintaud G, Noman M, Magdelaine-Beuzelin C, Ferrante $\mathrm{M}$, Degenne D, et al. Influence of trough serum levels and immunogenicity on long-term outcome of adalimumab therapy in Crohn's disease. Gastroenterology 2009;137:1628-40.

27. Zold E, Nagy A, Devenyi K, Zeher M, Barta Z. Successful use of adalimumab for treating fistulizing Crohn's disease with pyoderma gangrenosum: Two birds with one stone. World J Gastroenterol 2009; 15:2293-5.

28. Herfarth H, Obermeier F, Andus T, Rogler G, Nikolaus S, Kuehbacher $\mathrm{T}$, et al. Improvement of arthritis and arthralgia after treatment with infliximab (Remicade) in a German prospective, open-label, multicenter trial in refractory Crohn's disease. Am J Gastroenterol 2002;97:268890.

29. van der Heijde D, Kivitz A, Schiff MH, Sieper J, Dijkmans BA, Braun $\mathrm{J}$, et al. Efficacy and safety of adalimumab in patients with ankylosing spondylitis: results of a multicenter, randomized, double-blind, placebo-controlled trial. Arthritis Rheum 2006;54:2136-46.

30. Levine JB, Lukawski-Trubish D. Extraintestinal considerations in inflammatory bowel disease. Gastroenterol Clin North Am 1995; 24:633-46 